\title{
The first complete NLL BFKL study of Mueller Navelet jets at LHC
}

\section{Colferai}

Dipartimento di Fisica, Università di Firenze, Italy

INFN, Florence, Italy

Email: colferaiefi.infn.it

\section{F. Schwennsen}

Deusches Elektronen-Synchrotron DESY, Hamburg, Germany

Email: florian.schwennsen@desy.de

\section{Szymanowski}

Soltan Institute for Nuclear Studies, Warsaw, Poland \& CPHT, École Polytechnique, CNRS, 91128 Palaiseau Cedex, France

Email: lech.szymanowski@fuw.edu.pl

\section{S. Wallon*}

LPT, Université Paris-Sud, CNRS, 91405 Orsay, France \&

UPMC Univ. Paris 06, faculté de physique, 4 place Jussieu, 75252 Paris Cedex 05, France

E-mail: walloneth.u-psud.fr

\begin{abstract}
We report on the first next-to-leading BFKL study of the cross section and azimuthal decorrellation of Mueller Navelet jets. This includes next-to-leading corrections to the Green's function as well as next-to-leading corrections to the Mueller Navelet vertices. The obtained results for standard observables proposed for studies of Mueller Navelet jets show that both sources of corrections are of equal and big importance for final magnitude and final behavior of observables, in particular for the LHC kinematics investigated here in detail. The astonishing conclusion of our analysis is that the observables obtained within the complete next-lo-leading order BFKL framework of the present contribution are quite similar to the same observables obtained within next-to-leading logarithm DGLAP type treatment. The only noticeable difference is the ratio the azimuthal angular moments $\langle\cos 2 \varphi\rangle /\langle\cos \varphi\rangle$ which still differs in both treatments.
\end{abstract}

The 2011 Europhysics Conference on High Energy Physics-HEP 2011,

July 21-27, 2011

Grenoble, Rhône-Alpes France

\footnotetext{
*Speaker.
} 


\section{Introduction}

The high energy regime of QCD is one of the key questions of particle physics. In the semihard regime of a scattering process in which $s \gg-t$, logarithms of the type $\left[\alpha_{s} \ln (s /|t|)\right]^{n}$ have to be resummed, giving the leading logarithmic (LL) Balitsky-Fadin-Kuraev-Lipatov (BFKL) [1] Pomeron contribution to the gluon Green's function describing the $t$-channel exchange. To reveal this effect, various tests have been proposed in inclusive [2], semi-inclusive [3] and exclusive processes [4]. The basic idea is to select specific observables minimizing usual collinear logarithmic effects à la DGLAP [5] with respect to the BFKL one: the involved transverse scales should thus be of similar order of magnitude. We here consider the Mueller Navelet jets [6] in hadron-hadron colliders, defined as being separated by a large relative rapidity, while having two similar transverse energies. In a DGLAP scenario, an almost back-to-back emission is expected, while the allowed BFKL emission of partons between these two jets leads in principle to a larger cross-section, with a reduced azimuthal correlation between them. We review results of Ref. [7] where both the NLL Green function [8] and the NLL result for the jet vertices [9] are taken into account.

\section{NLL calculation}

The two hadrons collide at a center of mass energy $\sqrt{s}$ producing two very forward jets, whose transverse momenta are labeled by Euclidean two dimensional vectors $\mathbf{k}_{J, 1}$ and $\mathbf{k}_{J, 2}$, while their azimuthal angles are noted as $\phi_{J, 1}$ and $\phi_{J, 2}$. The jet rapidities $y_{J, 1}$ and $y_{J, 2}$ are related to the longitudinal momentum fractions of the jets via $x_{J}=\frac{\left|\mathbf{k}_{J}\right|}{\sqrt{s}} e^{y_{J}}$. We restrict ouselves to fixed rapidities and transverse momenta. For large $x_{J, 1}$ and $x_{J, 2}$, collinear factorization leads to

$$
\frac{\mathrm{d} \sigma}{\mathrm{d}\left|\mathbf{k}_{J, 1}\right| \mathrm{d}\left|\mathbf{k}_{J, 2}\right| \mathrm{d} y_{J, 1} \mathrm{~d} y_{J, 2}}=\sum_{\mathrm{a}, \mathrm{b}} \int_{0}^{1} \mathrm{~d} x_{1} \int_{0}^{1} \mathrm{~d} x_{2} f_{\mathrm{a}}\left(x_{1}\right) f_{\mathrm{b}}\left(x_{2}\right) \frac{\mathrm{d} \hat{\sigma}_{\mathrm{ab}}}{\mathrm{d}\left|\mathbf{k}_{J, 1}\right| \mathrm{d}\left|\mathbf{k}_{J, 2}\right| \mathrm{d} y_{J, 1} \mathrm{~d} y_{J, 2}},
$$

where $f_{\mathrm{a}, \mathrm{b}}$ are the parton distribution functions (PDFs) of a parton a (b) in the according proton. The resummation of logarithmically enhanced contributions are included through $k_{T}$-factorization:

$$
\frac{\mathrm{d} \hat{\sigma}_{\mathrm{ab}}}{\mathrm{d}\left|\mathbf{k}_{J, 1}\right| \mathrm{d}\left|\mathbf{k}_{J, 2}\right| \mathrm{d} y_{J, 1} \mathrm{~d} y_{J, 2}}=\int \mathrm{d} \phi_{J, 1} \mathrm{~d} \phi_{J, 2} \int \mathrm{d}^{2} \mathbf{k}_{1} \mathrm{~d}^{2} \mathbf{k}_{2} V_{\mathrm{a}}\left(-\mathbf{k}_{1}, x_{1}\right) G\left(\mathbf{k}_{1}, \mathbf{k}_{2}, \hat{s}\right) V_{\mathrm{b}}\left(\mathbf{k}_{2}, x_{2}\right),
$$

where the BFKL Green's function $G$ depends on $\hat{s}=x_{1} x_{2} s$. The jet vertices $V_{a, b}$ were calculated at NLL order in Ref. [9]. Combining the PDFs with the jet vertices one writes

$$
\begin{gathered}
\frac{\mathrm{d} \sigma}{\mathrm{d}\left|\mathbf{k}_{J, 1}\right| \mathrm{d}\left|\mathbf{k}_{J, 2}\right| \mathrm{d} y_{J, 1} \mathrm{~d} y_{J, 2}}=\int \mathrm{d} \phi_{J, 1} \mathrm{~d} \phi_{J, 2} \int \mathrm{d}^{2} \mathbf{k}_{1} \mathrm{~d}^{2} \mathbf{k}_{2} \Phi\left(\mathbf{k}_{J, 1}, x_{J, 1},-\mathbf{k}_{1}\right) G\left(\mathbf{k}_{1}, \mathbf{k}_{2}, \hat{s}\right) \Phi\left(\mathbf{k}_{J, 2}, x_{J, 2}, \mathbf{k}_{2}\right), \\
\text { where } \\
\Phi\left(\mathbf{k}_{J, 2}, x_{J, 2}, \mathbf{k}_{2}\right)=\int \mathrm{d} x_{2} f\left(x_{2}\right) V\left(\mathbf{k}_{2}, x_{2}\right) .
\end{gathered}
$$

In view of the azimuthal decorrelation we want to investigate, we define the coefficients

$\mathscr{C}_{m} \equiv \int \mathrm{d} \phi_{J, 1} \mathrm{~d} \phi_{J, 2} \cos \left(m\left(\phi_{J, 1}-\phi_{J, 2}-\pi\right)\right) \int \mathrm{d}^{2} \mathbf{k}_{1} \mathrm{~d}^{2} \mathbf{k}_{2} \Phi\left(\mathbf{k}_{J, 1}, x_{J, 1},-\mathbf{k}_{1}\right) G\left(\mathbf{k}_{1}, \mathbf{k}_{2}, \hat{s}\right) \Phi\left(\mathbf{k}_{J, 2}, x_{J, 2}, \mathbf{k}_{2}\right)$,

from which one can easily obtain the differential cross section and azimuthal decorrelation as

$$
\frac{\mathrm{d} \sigma}{\mathrm{d}\left|\mathbf{k}_{J, 1}\right| \mathrm{d}\left|\mathbf{k}_{J, 2}\right| \mathrm{d} y_{J, 1} \mathrm{~d} y_{J, 2}}=\mathscr{C}_{0} \quad \text { and } \quad\langle\cos (m \varphi)\rangle \equiv\left\langle\cos \left(m\left(\phi_{J, 1}-\phi_{J, 2}-\pi\right)\right)\right\rangle=\frac{\mathscr{C}_{m}}{\mathscr{C}_{0}}
$$


The guiding principle of the calculation is then to use the LL-BFKL eigenfunctions

$$
E_{n, v}\left(\mathbf{k}_{1}\right)=\frac{1}{\pi \sqrt{2}}\left(\mathbf{k}_{1}^{2}\right)^{i v-\frac{1}{2}} e^{i n \phi_{1}},
$$

although they strictly speaking do not diagonalize the NLL BFKL kernel. In the LL approximation,

$$
\begin{aligned}
& \mathscr{C}_{m}=\left(4-3 \delta_{m, 0}\right) \int \mathrm{d} v C_{m, v}\left(\left|\mathbf{k}_{J, 1}\right|, x_{J, 1}\right) C_{m, v}^{*}\left(\left|\mathbf{k}_{J, 2}\right|, x_{J, 2}\right)\left(\frac{\hat{s}}{s_{0}}\right)^{\omega(m, v)}, \\
& \text { where } \quad C_{m, v}\left(\left|\mathbf{k}_{J}\right|, x_{J}\right)=\int \mathrm{d} \phi_{J} \mathrm{~d}^{2} \mathbf{k} \mathrm{d} x f(x) V(\mathbf{k}, x) E_{m, v}(\mathbf{k}) \cos \left(m \phi_{J}\right),
\end{aligned}
$$

and $\omega(n, v)=N_{c} \alpha_{s} / \pi \chi_{0}\left(|n|, \frac{1}{2}+i v\right)$, with $\chi_{0}(n, \gamma)=2 \Psi(1)-\Psi\left(\gamma+\frac{n}{2}\right)-\Psi\left(1-\gamma+\frac{n}{2}\right)$. The master formulae of the LL calculation $(2.6,2.7)$ will also be used for the NLL calculation, the eigenvalue now turning to an operator containing a $v$ derivative, which acts on the impact factors and effectively leads to a contribution to the eigenvalue which depends on the impact factors.

At NLL, the jet vertices are intimately dependent on the jet algorithm [9]. We here use the cone algorithm. At NLL, one should also pay attention to the choice of scale $s_{0}$. We find the choice of scale $s_{0}=\sqrt{s_{0,1} s_{0,2}}$ with $s_{0,1}=\frac{x_{1}^{2}}{x_{J, 1}^{2}} \mathbf{k}_{J, 1}^{2}$ rather natural, since it does not depend on the momenta $\mathbf{k}_{1,2}$ to be integrated out. Besides, the dependence with respect to $s_{0}$ of the whole amplitude can be studied, when taking account the fact that both the NLL BFKL Green function and the vertex functions are $s_{0}$ dependent. In order to study the effect of possible collinear improvement [10], we have, in a separate study, implemented for $n=0$ the scheme 3 of the first paper of Ref. [10]. This is only required by the Green function since we could show by a numerical study that the jet vertices are free of $\gamma$ poles and thus do not call for any collinear improvement. In practice, the use of Eqs. $(2.6,2.7)$ leads to the possibility to calculate for a limited number of $m$ the coefficients $C_{m, v}$ as universal grids in $v$, instead of using a two-dimensional grid in $\mathbf{k}$ space. We use MSTW 2008 PDFs [11] and a two-loop strong coupling with a scale $\mu_{R}=\sqrt{\left|\mathbf{k}_{J, 1}\right| \cdot\left|\mathbf{k}_{J, 2}\right|}$. In order to compare our analysis with DGLAP NLO approaches [12] obtained through the NLL-DGLAP partonic generator DIJET [13], for which symmetric configurations lead to instabilities, we here display our results for $\left|\mathbf{k}_{J, 1}\right|=35 \mathrm{GeV},\left|\mathbf{k}_{J, 2}\right|=50 \mathrm{GeV}$ (see Ref. [7] for symmetric configurations).

\section{Results}

Fig. 1a and $1 \mathrm{~b}$ respectively display the cross-section and the azimuthal correlation as a function of the relative jet rapidity $Y$, for the LHC design center of mass energy $\sqrt{s}=14 \mathrm{TeV}$. This explicitely shows the dramatic effect of the NLL vertex corrections, of the same order as the one for the Green function [14]. In particular, the decorrelation based on our full NLL analysis is very small, similar to the one based on NLO DGLAP. The main source of uncertainties is due to the renormalization scale $\mu_{R}$ and to the energy scale $\sqrt{s_{0}}$. This is particularly important for the azimuthal correlation, which, when including a collinear improved Green's function, may exceed 1 for small $\mu_{R}=\mu_{F}$. The only remaining observable for which a noticeable difference can be expected between BFKL and DGLAP type of treatment is the ratio $\langle\cos 2 \varphi\rangle /\langle\cos \varphi\rangle$.

The NLL analysis presented here could be extended to describe forward jets production.

Work supported in part by the French-Polish Scientific Collaboration Agreement POLONIUM, the grant ANR-06-JCJC-0084 and by a PRIN grant (MIUR, Italy). 

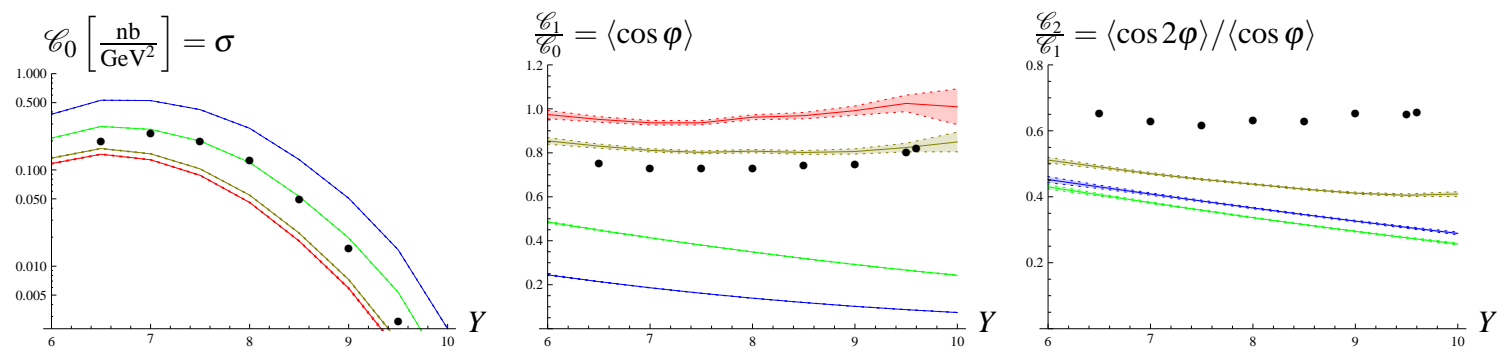

Figure 1: Differential cross section (a), azimuthal correlation $\langle\cos \varphi\rangle$ (b) and ratio $\langle\cos 2 \varphi\rangle /\langle\cos \varphi\rangle$ (c) in dependence on $Y$ for $\left|\mathbf{k}_{J, 1}\right|=35 \mathrm{GeV},\left|\mathbf{k}_{J, 2}\right|=50 \mathrm{GeV}$. The errors due to the Monte Carlo integration are given as error bands. Blue: pure LL result; Brown: pure NLL result; Green: combination of LL vertices with the collinear improved NLL Green's function; Red: full NLL vertices with the collinear improved NLL Green's function. Dots show the results of Ref. [12] obtained with DIJET [13].

\section{References}

[1] V. S. Fadin et al., Phys. Lett. B 60 (1975) 50; E. A. Kuraev et al., Sov. Phys. JETP 44 (1976) 443; Sov. Phys. JETP 45 (1977) 199; I. I. Balitsky and L. N. Lipatov, Sov. J. Nucl. Phys. 28 (1978) 822.

[2] A. J. Askew et al., Phys. Lett. B 325 (1994) 212; H. Navelet et al., Phys. Lett. B 385 (1996) 357; S. Munier et al., Nucl. Phys. B 524 (1998) 377; J. Bartels et al., Phys. Lett. B 389 (1996) 742; S. J. Brodsky et al., Phys. Rev. Lett. 78 (1997) 803; [Erratum-ibid. 79 (1997) 3544]; A. Bialas et al., Eur. Phys. J. C 2 (1998) 683; M. Boonekamp et al., Nucl. Phys. B 555 (1999) 540; J. Kwiecinski and L. Motyka, Phys. Lett. B 462 (1999) 203. S. J. Brodsky et al., JETP Lett. 70 (1999) 155; S. J. Brodsky et al., JETP Lett. 76 (2002) 249 [Pisma Zh. Eksp. Teor. Fiz. 76 (2002) 306].

[3] A. H. Mueller, Nucl. Phys. Proc. Suppl. 18C (1991) 125.

[4] I. F. Ginzburg et al., Nucl. Phys. B 284 (1987) 685; Nucl. Phys. B 296 (1988) 569; M. G. Ryskin, Z. Phys. C 57 (1993) 89; A. H. Mueller et al., Phys. Lett. B 284 (1992) 123. D. Y. Ivanov et al., Phys. Rev. D 58 (1998) 114026; Phys. Lett. B 478 (2000) 101 [Erratum-ibid. B 498 (2001) 295]. B. Pire et al., Eur. Phys. J. C 44 (2005) 545; R. Enberg et al., Eur. Phys. J. C 45 (2006) 759 [Erratum-ibid. C 51 (2007) 1015]; M. Segond et al., Eur. Phys. J. C 52 (2007) 93; D. Y. Ivanov and A. Papa, Nucl. Phys. B 732 (2006) 183; Eur. Phys. J. C 49 (2007) 947; F. Caporale et al., Eur. Phys. J. C 53 (2008) 525.

[5] V. N. Gribov et al., Sov. J. Nucl. Phys. 15 (1972) 438; L. N. Lipatov, Sov. J. Nucl. Phys. 20 (1975) 94; G. Altarelli et al., Nucl. Phys. B 126 (1977) 298; Y. L. Dokshitzer, Sov. Phys. JETP 46 (1977) 641.

[6] A. H. Mueller and H. Navelet, Nucl. Phys. B 282 (1987) 727.

[7] D. Colferai, F. Schwennsen, L. Szymanowski, S. Wallon, JHEP 1012 (2010) 026.

[8] V.S. Fadin and L.N. Lipatov, Phys. Lett. B 429 (1998) 127; M. Ciafaloni and G. Camici, Phys. Lett. B 430 (1998) 349.

[9] J. Bartels, D. Colferai, and G. P. Vacca, Eur. Phys. J. C 24 (2002) 83; 29 (2003) 235.

[10] G. P. Salam, JHEP 07 (1998) 019; M. Ciafaloni et al., Phys. Lett. B 452 (1999) 372; Phys. Rev. D 60 (1999) 114036; Phys. Rev. D 68 (2003) 114003.

[11] A. D. Martin, W. J. Stirling, R. S. Thorne and G. Watt, Eur. Phys. J. C 63 (2009) 189.

[12] M. Fontannaz, LPT-Orsay/09-86 (2009).

[13] P. Aurenche, R. Basu, and M. Fontannaz, Eur. Phys. J. C 57 (2008) 681.

[14] A. Sabio Vera et al., Nucl. Phys. B 776 (2007) 170; C. Marquet et al., Phys. Rev. D 79 (2009) 034028. 